\title{
The Optimal Power Flow Calculation of Power System Based on the Annealing Algorithm
}

\author{
Xiaodong Yu \\ North China Electric Power University, Changping District, Beijing, China \\ Email: 307426897@qq.com
}

Received April, 2013

\begin{abstract}
The result of OPF whose task is to compute the voltage and angle of each node in power system is the basic of stability calculation and failure analysis in power system. For this goal, the idea of simulated annealing method is introduced, mixed with the greedy randomized algorithm (GRASP), and then the hybrid SA algorithm is obtained. The algorithm is applied to the multi-objective optimal power flow calculation of power system, and the effectiveness of the algorithm given in this paper is verified by analysis of examples.
\end{abstract}

Keywords: Annealing Algorithm; Power System; Power Flow Calculation

\section{Introduction}

Optimal power flow was put forward by Carpenter of EDF in the early $60 \mathrm{~s}$, having attracted a large number of scholars to study this problem for years and been applied in multiple areas. Optimal power flow itself is a kind of multivariable, high-dimensional, multi-constrained mixed nonlinear optimization problem with coexistence of continuous and discrete variables in mathematics. At present, the solution of power system OPF can be divided into two categories. One is based on numerical solution, mainly including: linear programming, nonlinear programming, quadratic programming, decoupling method, Newton method and interior point method. This kind of algorithm has different features, which in general are in relatively fast computing speed, suitable for on-line calculation, but more troublesome in handling constraint conditions, especially discrete variables. The other is based on the method of artificial intelligence, such as: simulated annealing method, genetic algorithms, evolutionary programming method, Tabu search method, particle swarm optimization (pso) algorithm, chaotic search method, artificial immune algorithm, entropy theory method and so on. This kind of algorithm mainly imitates natural and biological features and behavior to deal with problems without complicated data processing and numerical calculation. The procedure is simple, but the calculation is relatively slow, not suitable for the modern large-scale power system online calculation and analysis. In addition, the fuzzy set theory and parallel computing method have already been applied to power system flow calculation, and combined with various algorithms above, it con- stantly enriches the content of optimal power flow algorithm.

\section{Simulated Annealing Algorithm}

In scientific and engineering computing, one common problem is to seek the minimal point of a non-linear function $\mathrm{f}(\mathrm{x})$ in $\mathrm{Rn}$ or on a bounded domain. When $\mathrm{F}(\mathrm{x})$ is derivative, a basic algorithm is the steepest descent method. This method is starting from a selected initial value, using the following formula for iteration, i.e.

$$
x_{n+1}=x_{n}-\eta_{n} \nabla f\left(x_{n}\right)
$$

$\nabla f$ means the function gradient, which is a parameter related to iterative steps. Taking proper values of this parameter ensures that each iteration makes the function value decrease. In addition, there are various algorithms of seeking minimal function. However, the traditional algorithms represented by the downhill method have common disadvantages that they do not guarantee to obtain a global minimum, but only to converge to a local minimum point determined by the initial value. And the simulated annealing algorithm is a good solution to this problem.

Simulated annealing algorithm is derived from the principle of solid annealing. Its core idea is very similar to the fundamental thermodynamic principle, especially similar to liquid flow and crystallization, as well as metal cooling and annealing. At high temperatures, a large number of molecules of the liquid move relatively free. If the liquid is cooled slowly, the thermal mobility will disappear. Large number of atoms can often arrange 
themselves in rows, forming a pure crystal, which in each direction is arranged orderly within the distance of millions of times of that between individual atoms. Thus, the essence of this process is cooling slowly to gain sufficient time for a large number of atoms to be redistributed prior to the loss of mobility. It is a necessary condition to ensure a lower energy state. In short, the physical annealing process consists of the following three components: 1) the heating process. Its purpose is to enhance the thermal motion of the particles, so that they can deviate from the equilibrium position. When the temperature is high enough, the solid will be melted into liquid, thereby eliminating the non-uniform state that may exist in the system originally, so that the subsequent cooling process can start from an equilibrium state. The melting process is related to the entropy increasing process of the system. And the energy of the system also increases with the increase of temperature. 2) The isothermal process. Physical knowledge tells us that for the closed system that exchanges heat with the surrounding environment but of constant temperature, the spontaneous change of the state of the system is always towards the free energy-decreased direction. The system reaches equilibrium state when the free energy decreases to the minimum. 3) the cooling process. Its purpose is to make the thermal motion of the particles weaken and gradually orderly. The energy of the system gradually declines, resulting in a low-energy crystal structure.

\subsection{The Basic Steps of Annealing Algorithm}

1) Randomly generates an initial solution $x 0$, make $\mathrm{xbest}=\mathrm{x} 0$, and calculate the objective function value $\mathrm{E}(\mathrm{x} 0)$;

2) Set the initial temperature $T(0)=T 0$, the number of iterations $\mathrm{i}=1$;

3) Do while $T(\mathrm{i})>\mathrm{Tmin}$

1) for $\mathrm{j}=1 \sim \mathrm{k}$

2) For the current optimal solution xbest, according to a certain neighborhood function, generate a new solution xnew. Calculate:

Table 1. he similar relationship between simulated annealing algorithm and the physical annealing process.

\begin{tabular}{ll}
\hline Simulated annealing & Physical annealing \\
\hline solution & particle state \\
optimal solution & energy minimum \\
Set the initial temperature & dissolution process \\
Metropolis sampling process & isothermal process \\
the decrease of control parameter & cooling \\
objective function & energy \\
\hline
\end{tabular}

New objective function value $\mathrm{E}(\mathrm{xnew})$, and calculate the increment of the objective function value

$\Delta \mathrm{E}=\mathrm{E}(\mathrm{xnew})-\mathrm{E}$ (xbest) 。

3) If $\Delta E<0$, xbest $=$ xnew;

4) If $\Delta \mathrm{E}>0, \quad \mathrm{p}=\exp (-\Delta \mathrm{E} / \mathrm{T}(\mathrm{i}))$;

1) if $\mathrm{c}=\operatorname{random}[0,1]<\mathrm{p}, \quad \mathrm{xbest}=\mathrm{xnew}$; otherwise $\mathrm{xbest}=\mathrm{xbest}$ 。

5) End for

6) $\mathrm{i}=\mathrm{i}+1$;

7) End Do

8) Output the current optimal point, this is the end of calculation.

\subsection{The Requirements and Application Scope of Annealing Algorithm}

Annealing algorithm is a soft computing method which has attracted increasing attention in recent years. It can solve some of the problems that are difficult for traditional nonlinear programming method to solve, and has been widely studied in VLSI, generation scheduling, control engineering, machine learning, neural networks, image processing, function optimization and many other fields.

The application of annealing algorithm must meet the following requirements of three aspects:

1) The concise description of the problem, namely the mathematical model, consists of three parts: the solution space, the objective function and the initial solution;

a. The solution space is the set of all possible (feasible or infeasible) solutions of the problem, which defines the scope of the selection of the initial solution and the generation of new solutions. For unconstrained optimization problems, the solution space is the set of all feasible solutions, while in many combinatorial optimization problems, and the solution need not only meet the optimization of the objective function, but also meet a set of constraints. Therefore, the solution set may contain some infeasible solutions. $b$. The objective function is the mathematical description of the optimization goal of the problem, usually expressed as a sum formula of certain optimization goals. The selection of the objective function must correctly reflect the overall optimization requirements of the problem. In general, the objective function is not necessarily the optimal objective function value of the problem, but the correspondence relationship is obvious. In addition, the objective function should be easy to calculate. It will be beneficial to simplify the calculation of the objective function in the optimization process and easy to improve the efficiency of the algorithm. c. The initial solution is the starting point of the beginning of the algorithm iteration. The selection of the initial solution should make the algorithm derive the better final solution. But a large number of test results show 
that the simulated annealing algorithm is a "robust" algorithm, i.e. the final solution of the algorithm has little dependence on the initial solution.

2) The generation of the new solution and the acceptance mechanism;

a. A generating device generates a new solution in the solution space starting from the current solution, to facilitate the calculations and acceptance below. And usually the selecting method that generates the new solution goes through a simple transformation of the current solution, such as the replacement, exchange or inversion of all or part of elements constituting the solution. The method of the generation of new solution determines the neighborhood structure of the current solution. b. Calculate the difference of the objective function accompanied by the new solution. Because the difference of the objective function is only generated by converting part, the difference of the objective function is better calculated as the increment. c. To determine whether the new solution is accepted. Judgment is based on the Metropolis criterion. In addition, in the constrained combinatorial optimization problem, the judgment of the feasibility of the new solution should be added to this acceptance criterion. d. When the new solution is determined to accept, replace the current solution with the new solution, meanwhile amend the objective function value. In this case, the current solution achieves an iteration. The next round of tests can begin on this basis. When the new solution is judged to be discarded, continue the next round of tests on the basis of the original solution[1].

3) The cooling schedule.

The so-called cooling schedule is a set of parameters of control algorithm process used to approximate the gradual convergence state of the simulated annealing algorithm, making the algorithm return to an approximate optimal solution after the execution process in the limited time. Cooling schedule includes the initial value of the control parameter, its attenuation function, corresponding length of the Markov chain and the stop criterion. It is an important factor affecting the test performance of the simulated annealing algorithm and its reasonable selection is crucial to the application of the algorithm[2].

\section{The Application of the Annealing Algorithm in Power System Flow Calculation}

\subsection{Annealing Algorithm}

Simulated Annealing (SA) is a universal random search algorithm, first proposed by Metropolis et al in 1953, but not causing repercussions. Until 1983, Kirkpatrick et al put forward modern SA algorithm, and successfully used it to solve large-scale combinatorial optimization prob- lems. Thanks to the advantages of SA algorithm such as overcoming the dependence on initial values and avoiding partial optimization, it has been widely used in various fields.

The basic idea of the SA algorithm is derived from the annealing process in physics: First, give an initial high temperature. Beginning with this temperature, use the Metropolis sampling strategy with probabilistic jumping characteristics to search randomly in the solution space. Repeat the sampling process as the temperature drops, and ultimately obtain the global optimal solution. The key technology of the SA algorithm is the Metropolis sampling strategy, which sets the criteria and methods of the current solution moving to other solutions. Set $X$ as the current solution, $X^{\prime}$ as a new solution in the neighborhood area, and their increment of the target value as: $\Delta f=f\left(X^{\prime}\right)-f(X)$. When seek the minimum value, if $\Delta f<0$, unconditionally move to a new state, i.e. $X^{\prime}$; otherwise, according to certain probability $p$ ( $p=\exp \left(-\Delta f / T_{k}\right)$, wherein $T_{k}$ is the current temperature), determine whether to move to a new state, and provided that $q=\operatorname{rand}(0,1)$, if $\mathrm{p}>\mathrm{q}$, then move to a new state. The Metropolis criterion is the assurance of SA algorithm to jump out of local extreme value.

The entire computing process of SA algorithm includes two loops, the inner loop and outer loop. The inner loop is local search process controlled by the Metropolis sampling criterion; the outer loop is temperature dropping process controlled by cooling function. The common cooling function has the following two kinds:

1) $T_{k+1}=T_{k} \cdot r$, where $r \in(0.95,0.99)$, the greater $r$ is, the more slowly the temperature drops. This method is simple and practicable. The temperature drops at the same ratio every time.

2) $T_{k+1}=T_{k}-\Delta T, \Delta T$ is the step size that the temperature decreases every time. This method can simply control the total number of times of the temperature drop, and every time the size that the temperature drops is equal.

The specific realization method of SA algorithm solving the problem of transmission network planning is as follows:

1) Randomly generate an initial program $X$, set the initial temperature as $T=T_{0}$ and the final temperature as $T=T_{f}$, the iteration frequency $\mathrm{k}=1, T_{k}=T_{0}$.

2) According to certain rules, generate the new program $X^{\prime}$, calculate the increment of the target value $\Delta f=f\left(X^{\prime}\right)-f(X)$.

3) If $\Delta f<0$, move from the current program to the new program, go to step (4); otherwise generate $q=\operatorname{rand}(0,1)$, make $p=\exp \left(-\Delta f / T_{k}\right)$, if $\mathrm{p}>\mathrm{q}$, then move to the new program.

4) If it reaches thermal equilibrium (the number of inner loops reaches maximum), go to step (5), otherwise go 
to step (2).

(5) Decrease $T_{k+1}=T_{k}-\Delta T, \mathrm{k}=\mathrm{k}+1$, if $T_{k}<T_{f}$, the algorithm stops; otherwise go to step (2).

Although SA algorithm can avoid local optimum, it has the following two drawbacks: 1) the initial solution of the SA algorithm is randomly generated. If the quality of the initial solution is too poor, it will affect the search performance of the algorithm. 2) SA algorithm belongs to a single point of optimization, and the optimization process is time-consuming.

\subsection{The Hybrid Annealing Algorithm}

According to the advantages and disadvantages of the GRASP algorithm and SA algorithm, design a hybrid SA algorithm: the introduction of Metropolis sampling strategy of the SA algorithm in the local search process makes the hybrid SA algorithm accept inferior solutions with probability out the local search phase, thereby enhancing the ability of the algorithm jumping out of local maximum. To further improve the computing performance of the hybrid SA algorithm, improve the algorithm from the following two aspects with the GRASP algorithm:

1) Have parallel processing of the algorithm, namely generate $\mathrm{S}$ initial feasible solutions in the construction phase and bring them together with local search $[3]$.

2) Introduce the judge selection mechanism, the mathematical expression is as follows:

$$
f(x) \leq(1+r) \cdot \min , r>0
$$

where min is target value of the current optimal program; $r$ is the filter coefficient. Before the local search in the current solution each time, first judge its function value, only when the function value meets the formula above, then have the local search, which can greatly improve the convergence speed.

The specific steps of Hybrid SA algorithm applied to the transmission grid planning are as follows:

1) Initialize the algorithm parameter: set the population number as $\mathrm{S}$, the initial temperature as $T_{k}$, the end temperature as $T_{f}$, the step size of temperature drop step as $\Delta T$, the filter coefficient as $\mathrm{r}$.

2) Use the GRASP algorithm to construct $S$ initial feasible solutions, and work out the target value of the current optimal solution as min.

3) At the current temperature, implement the local search process of the GRASP algorithm. And when searching, the Metropolis criterion accepts inferior solutions with probability, to update the position of the current solution. After all solutions perform local search, update the current optimal solution min.

4) Analyze whether min has improvement or not in the $\mathrm{n} 1$ consecutive iteration. If there is no improvement, go to step (6). Otherwise, to determine whether the current temperature is lower than the end temperature, if lower, go to step (6).

5) Make $\mathrm{k}=\mathrm{k}+1, T_{k+1}=T_{k}-\Delta T$, go to step (3).

6) Output the current optimal solution as the optimal solution, stop the calculation.

Hybrid SA algorithm flow chart is shown in Figure 1.

\section{Example and Explanation}

\subsection{Example and Solution}

Node (1) is the balanced node, $\mathbf{U}_{\mathbf{1}}=\mathbf{1} \angle \mathbf{0}^{\circ}$, node (2) is

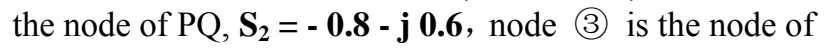
$\mathrm{PV}, \mathbf{P}_{3}=\mathbf{0 . 4}, \mathbf{U}_{3}=\mathbf{1 . 1}$, the network structure and parameters are shown in Figure $\mathbf{1}$ ( the parameters mean the per-unit value of the admittance), and find the result of the load flow calculation.

The steps of using simulate anneal arithmetic to conduct the load flow calculation for the system of the three simple nodes shown in the figure $\mathbf{2}$ are as follows:

1) Generate branched impedance matrix $Y[i, j]$ and node-to-ground capacitance matrix.

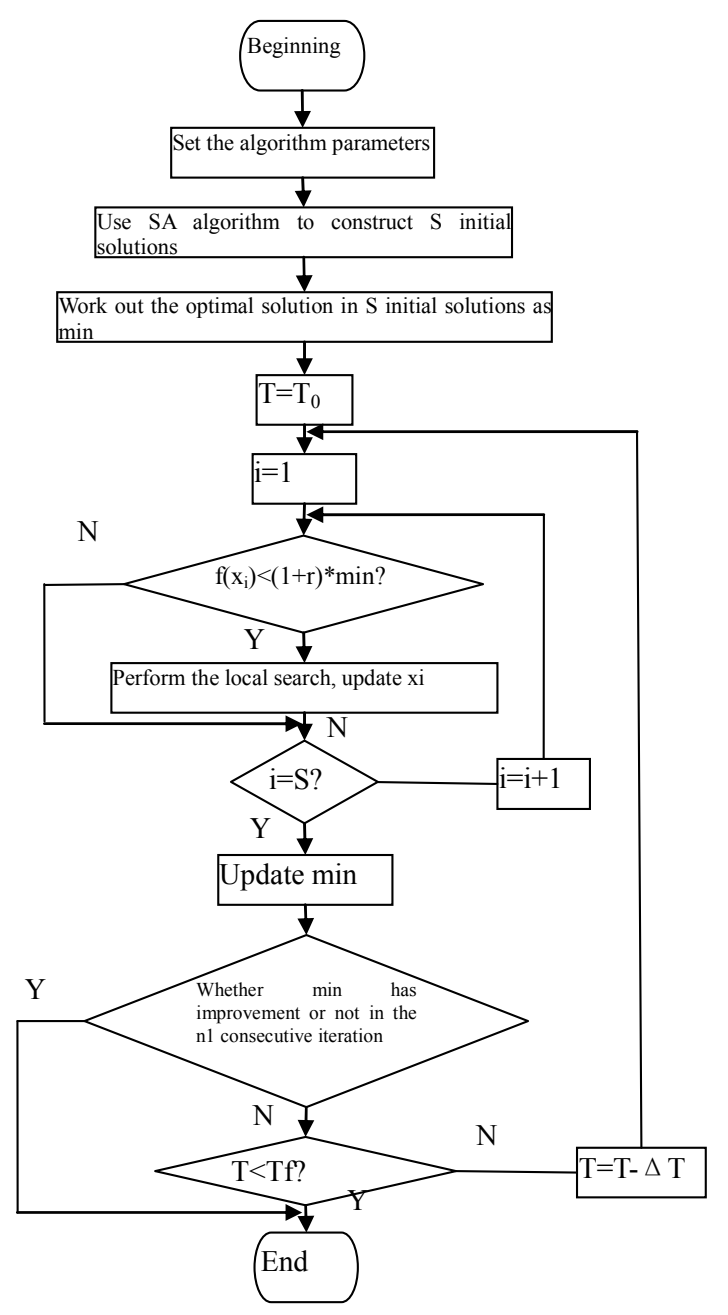

Figure 1. SA algorithm flow. 


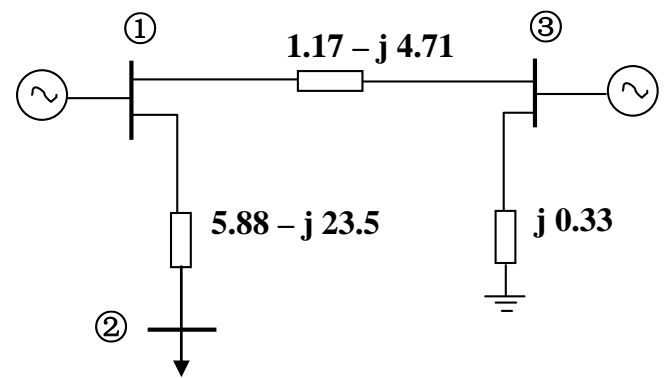

Figure 2. Network structure of the example 1.

2) Generate the solution space Sline1[i] and Sline2[i], Sline1[i] and Sline2[i] store the active power and the reactive power which flow through the starting end of the circuit branch respectively, and give a proper initial value.

3) Set the initial temperature of simulate anneal arithmetic as $\mathrm{T}=1$, temperature drop coefficient at $=0.999$, final temperature $\mathrm{TF}=\mathrm{e}^{\wedge}-30$, iterative coefficient $\mathrm{k}=1$.

4) Calculate the voltage of node 2 and node 3 , and the load power of the node 2 .

$$
\begin{aligned}
d \dot{U} & =\frac{P R+Q X}{U}+j \frac{P X-Q R}{U} \\
\Delta S_{l} & =\frac{P^{2}+Q^{2}}{U^{2}} R+j \frac{P^{2}+Q^{2}}{U^{2}} X \\
\Delta S_{c} & =-j \frac{1}{2} B U^{2}
\end{aligned}
$$

5) Get the difference value between the voltage of node 2 and the voltage given by the system as the $\Delta f$, similarly for the voltage of node 3 , and get the difference value between the load power of node 2 and the power given by the system. And judge if the values of the element in $\Delta f$ are all 0 , if the values of the element in $\Delta f$ are all 0 , then turn to step 7 , if the values of the element in $\Delta f$ are not all 0 , then use Metropolis standard to judge the element.

6) For these elements which are accepted by Metropolis standard, keep the solved value as the new value; for these elements which are not accepted by Metropolis standard, compare the solved value with the value given by the system, and based on the result of the comparison to disturb, at that moment, $\mathrm{k}=\mathrm{k}+1, \mathrm{~T}=\mathrm{T} *$ at, if $\mathrm{T}<\mathrm{TF}$, then turn to step 7, otherwise turn to step 4 .

7) Make the solved value as the final conclusion, and further get the voltage and the network loss value of each node in the whole network.

The results calculated by simulate anneal arithmetic are as follows table 1 and table 2.

The whole network loss: Ploss $=0.0258944$ Qloss $=$ 0.103945

The results calculated by Newton-Raphson method are as follows table 3 and table $\mathbf{4}$ :
Table 1. Voltage and phase angle of each node computed by SA.

\begin{tabular}{cccccc}
\hline $\begin{array}{c}\text { Node } \\
\text { no. }\end{array}$ & voltage & $\begin{array}{c}\text { Phase angle of } \\
\text { node voltage } \\
\text { (radian) }\end{array}$ & $\begin{array}{c}\text { Phase angle of } \\
\text { node voltage } \\
\text { (angle) }\end{array}$ & $\begin{array}{c}\text { Power } \\
\text { Injected } \\
\text { in node }\end{array}$ & $\begin{array}{c}\text { No power } \\
\text { Injected } \\
\text { in node }\end{array}$ \\
\hline 1 & 1 & 0 & 0 & 0.43 & 0.25 \\
2 & 0.968 & -0.027 & -1.5 & -0.8 & -0.6 \\
3 & 1.1 & 0.0524 & 2.9 & 0.4 & 0.46 \\
\hline
\end{tabular}

Table 2. Power flow of the circuit branch computed by SA.

\begin{tabular}{ccccc}
\hline $\begin{array}{c}\text { Circuit } \\
\text { branch no. }\end{array}$ & $\begin{array}{c}\text { Left } \\
\text { node }\end{array}$ & $\begin{array}{c}\text { Right } \\
\text { node }\end{array}$ & Begin & End \\
\hline 1 & 1 & 3 & $-0.38+\mathrm{j}-0.4$ & $0.4+\mathrm{j} 0.46$ \\
2 & 1 & 2 & $0.82+\mathrm{j} 0.65$ & $-0.8+\mathrm{j}-0.6$ \\
3 & 3 & 0 & $0+\mathrm{j}-0.4$ & $0+\mathrm{j} 0$ \\
\hline
\end{tabular}

Table 3. Voltage and phase angle of each node computed by Newton-Raphson.

\begin{tabular}{cccccc}
\hline $\begin{array}{c}\text { Node } \\
\text { no. }\end{array}$ & $\begin{array}{c}\text { Voltage } \\
\text { of the } \\
\text { node }\end{array}$ & $\begin{array}{c}\text { Phase angle of } \\
\text { node voltage } \\
\text { (radian) }\end{array}$ & $\begin{array}{c}\text { Phase angle of } \\
\text { node voltage } \\
\text { (angle) }\end{array}$ & $\begin{array}{c}\text { Power } \\
\text { Injected } \\
\text { in node }\end{array}$ & $\begin{array}{c}\text { No power } \\
\text { Injected } \\
\text { in node }\end{array}$ \\
\hline 1 & 1 & 0 & 0 & 0.425894 & 0.245894 \\
2 & 0.96653 & -0.0269036 & -1.54146 & -0.8 & -0.6 \\
3 & 1.1 & 0.0520552 & 2.98255 & 0.4 & 0.45805 \\
\hline
\end{tabular}

Table 4. Power flow of the circuit branch computed by Newton-Raphson.

\begin{tabular}{ccccc}
\hline $\begin{array}{c}\text { Circuit } \\
\text { no. }\end{array}$ & $\begin{array}{c}\text { Left } \\
\text { node }\end{array}$ & $\begin{array}{c}\text { Right } \\
\text { node }\end{array}$ & Begin & End \\
\hline 1 & 1 & 3 & $-0.38481+\mathrm{j}-0.396925$ & $0.4+\mathrm{j} 0.45805$ \\
2 & 1 & 2 & $0.810705+\mathrm{j} 0.642818$ & $-0.8+\mathrm{j}-0.600001$ \\
3 & 3 & 0 & $0+\mathrm{j}-0.3993$ & $0+\mathrm{j} 0$ \\
\hline
\end{tabular}

The whole network loss: Ploss $=0.0258944$ Qloss $=$ 0.103945

\subsection{Some Explanations and Comparison}

1) Temperature $T_{k}$

The Metropolis criterion is the key factor for hybrid SA algorithm to have global search, ensuring that the algorithm has the ability of jumping out from the local minimum and inclining to the global optimum. The size of the temperature $T_{k}$ is the core of the Metropolis criterion: when $T_{k}$ is very large, the algorithm will have a wide area search, and will accept any solution in the neighborhood area, even if this solution is inferior to the current solution; when $T_{k}$ is very small, the algorithm 
will have a local search, and only accept the better solution in the current neighborhood area. Thus, the selection of $T_{k}$ is also very important. It directly affects the convergence of the algorithm.

2) The temperature coefficient $\alpha$ and the annoyance value

The Metropolis criterion is the key factor for hybrid SA algorithm to have a proper rate of convergence. If $\alpha$ is too large, it is possible to reach the solution when the temperature is still high. In this case, the computation source is wasted. If $\alpha$ is too small, the final solution will not be a optimal one. For the annoyance value, it is also significant to choose a appropriate value. A large value may cause a misconvergence with a strict accuracy requirement and a small value leads to a slow rate of convergence. In order to obtain an excellent convergence performance, the value of $\alpha$ and the annoyance value should be coordinate to reach a balance. To obtain better convergence property, the value of $\alpha$ and the annoyance value also can be set as a function of deviation value $\Delta f[4]$.

3) The effectiveness of the method

As Newton-Raphson method is still the main means of calculating the load flow of the electrical power system with its calculated results having very big referential significances, while the simulate anneal arithmetic is very similar with the Newton-Raphson method, so we can say that use simulate anneal arithmetic is efficient for calculating the load flow of the electrical power system.

\section{Conclusions}

This paper has a deep analysis of the characteristics of the SA algorithm, and combines GRASP algorithm with
SA algorithm to form hybrid SA algorithm. The hybrid algorithm gives full play to the advantages of the two original algorithms. It makes the SA algorithm of high computing efficiency greatly improved on the convergence performance by introducing the Metropolis sampling criterion of the SA algorithm into GRASP algorithm. According to the characteristics of the transmission network planning problem, in order to reduce the amount of calculation, the hybrid SA algorithm has taken local improvement, introducing screening mechanism, which makes the optimal solution have local search, and further improves the calculation speed of the algorithm. In this way the annealing algorithm is applied to solve the transmission network planning problem, and its feasibility is verified and superiority by examples.

\section{REFERENCES}

[1] W. D. Rosehart, C. A. Caniares and V. H. Quintana, "Multi-ob-jective Optimal Power Flow to Evaluate Voltage Sedcurity Costs in Power Networls," IEEE Transactions on Power Systems, Vol. 18, No. 2, 2003, pp. 578-587. doi:10.1109/TPWRS.2003.810895

[2] J. A. Momoh, M. E. EI-Hawary and R. Adapa, "Nonlinear and Quadratic Programming Approaches, Part I," A Review of Selected Optimal Power Flow Literature to 2003, Vol. 14, No. 1, 2005, pp. 96-104.

[3] A. G. Bakirtzis, et al., "Optimal Power Flow by Enhanced Genetic Algorithm," IEEE Transactions on Power Systems, 2008, Vol. 17, No. 2, pp. 229-236. doi:10.1109/TPWRS.2002.1007886

[4] X. Zhang, R. W. Dunn and F. Li, "Stability Constrained Optimal Power Flow for the Balancing Market Using Genetic A lgorithms," Proceedings of IEEE PES General Meeting, Toronto(Canada):2003. 